\title{
Effect of gestational smoke exposure on atopic dermatitis in the offspring
}

\author{
Wang I-J, Hsieh W-S, Wu K-Y, Guo YL, Hwang Y-H, Jee S-H, Chen \\ $\mathrm{P}$-C. Effect of gestational smoke exposure on atopic dermatitis in the \\ offspring. \\ Pediatr Allergy Immunol 2008: 19: 580-586. \\ (C) 2008 The Authors \\ Journal compilation @ 2008 Blackwell Munksgaard
}

The adverse impact of smoking on respiratory diseases and birth outcomes in children is well-known. However, the influence of smoke exposure including environmental tobacco smoke (ETS) and maternal smoking during pregnancy on atopic dermatitis (AD) is not clear. The purpose of this study was to evaluate the effect of gestational smoke exposure on the development of $\mathrm{AD}$ in the offspring on the basis of the maternal and cord blood cotinine. We recruited 261 mother and newborn pairs in 2004. Cord blood and information on perinatal factors of children were gathered at birth. At $2 \mathrm{yr}$ of age, information about development of $\mathrm{AD}$ and environmental exposures were collected. We compared $\mathrm{AD}$ with non-AD children for the concentration of cotinine in cord and maternal blood measured by high performance liquid chromatography-mass spectrometry. Multiple logistic regressions were performed to estimate the relationship of cotinine levels and AD. About 150 mother and child pairs completed the follow-up study and specimen collection with $38(25.3 \%)$ children developing AD. Two (1.3\%) out of 150 mothers smoked during pregnancy, while $38(25.3 \%)$ mothers reported having ETS exposure. Cotinine levels in cord blood and maternal blood were highly correlated $(\mathrm{r}=0.71, \mathrm{p}<0.001)$. The risk of $\mathrm{AD}$ was found to increase with maternal and cord blood cotinine levels in a dose-response manner ( $\mathrm{p}$ for trend $=0.01$ ). Children exposed to high levels ( $>75$ th percentile) had a significantly increased risk of AD. Smoke exposure during pregnancy might increase the risk of $\mathrm{AD}$ in children. Avoidance of prenatal smoke exposure may be warranted for early prevention.

\author{
I-Jen Wang ${ }^{1,2}$, Wu-Shiun Hsieh ${ }^{3}$, \\ Kuen-Yuh $\mathbf{W u}^{4}$, Yueliang Leon Guo ${ }^{5}$ \\ Yaw-Huei Hwang ${ }^{2}$, Shiou-Hwa Jee ${ }^{6}$ \\ and Pau-Chung Chen ${ }^{2}$ \\ ${ }^{1}$ Department of Pediatrics, Taipei Hospital, \\ Department of Health, Taipei, ${ }^{2}$ Institute of \\ Occupational Medicine and Industrial Hygiene, \\ College of Public Health, National Taiwan University, \\ Taipei, ${ }^{3}$ Department of Pediatrics, National Taiwan \\ University Hospital and National Taiwan University \\ College of Medicine, Taipei, ${ }^{4}$ Division of \\ Environmental Health and Occupational Medicine, \\ National Health Research Institutes, Miaoli, \\ ${ }^{5}$ Department of Environmental and Occupational \\ Medicine, National Taiwan University Hospital and \\ National Taiwan University College of Medicine, \\ Taipei, ${ }^{6}$ Department of Dermatology, National Taiwan \\ University Hospital and National Taiwan University \\ College of Medicine, Taipei, Taiwan
}

Key words: atopic dermatitis; cotinine; environmental tobacco smoke

Professor Pau-Chung Chen, Institute of Occupational Medicine and Industrial Hygiene, College of Public Health, National Taiwan University, 17 Syujhou Road, Taipei 10055, Taiwan

Tel.: +886 233228088

Fax: +886 223582402

E-mail: pchen@ntu.edu.tw

Accepted 2 May 2008
Most studies evaluating the effect of smoking and environmental tobacco smoke (ETS) exposure on health outcomes rely on questionnaires $(1,2)$. Self-reported measures, such as number of cigarettes smoked per day in smokers or hours per day exposed to ETS by non-smokers, are highly imprecise because of individual differences in smoking habits and environmental characteristics $(3,4)$. ETS exposure might change with the number of cigarettes smoked, proximity of nonsmokers to smokers, room ventilation and other environmental characteristics as well as individual differences concerning the adverse effects of
ETS. At present, cotinine measured in blood, saliva, or urine is considered to be the most specific and the most sensitive biomarker for smoke exposure (5). Cotinine, the major metabolite of nicotine, has a longer half life (18-20 h) than nicotine (1-2 h) (6). Cord serum cotinine concentration is related to daily smoking rate during pregnancy (7). It appears to be the most adequate biomarker of fetal exposure to smoking at the end of pregnancy, distinguishing not only active smoking from passive smoking but also exposure to ETS from non-exposure (8). To avoid reporting bias or misclassification of 
exposure and non-exposure, we based the calculation of smoking associated health risks on the concentrations of the plasma cotinine as an objective biomarker.

In the Taiwan 2001 survey, $4.5 \%$ reproductive age women smoke while $57-59 \%$ of non-smoking women of childbearing ages were exposed to ETS (9). Unborn children are at risk from cigarette smoke, either from the mother smoking or from ETS. They can be underweight at birth and have decreased lung function (10). In contrast to low birth weight and respiratory tract diseases, there is no consist association between smoke exposure and atopic dermatitis (AD) in children. Krämer et al. reported that school beginners were at a higher risk of developing $\mathrm{AD}$ when exposed to ETS (11). Maternal smoking during pregnancy or lactation increased the risk of the offspring developing $\operatorname{AD}(12,13)$ while some studies revealed negative relationships (14-18).

As AD causes significant family stress and healthcare expenditures, identification of risk factors for early prevention is of critical importance. Therefore, in this study, we will investigate if ETS exposure and maternal active smoking during pregnancy will increase the risk of AD on the basis of the cord and maternal blood cotinine.

\section{Methods}

Study population

Considering potential environmental exposures and nationwide representativeness, we recruited our subjects from medical centers, regional hospitals, local hospitals, and clinics in Taiwan in 2004. Pregnant women during the third trimester of pregnancy, who had prenatal examination in selected hospitals, were invited to join in this study. Cord blood was collected at delivery. A total of 261 mother and child pairs were recruited with informed consents and the study was approved by the Joint Institution Review Board in Taiwan.

Cases of AD were defined by International Study of Asthma and Allergies in Childhood (ISAAC) questionnaire through all of the three questions 'Has your child ever had an itchy rash which was coming and going for at least 6 months at any time?', 'Has the itchy rash been coming and going over elbows, knees, face, wrists, or generalized (four or more localizations)?', and 'Has your child ever had AD diagnosed by a doctor?'. As described elsewhere, a dermatologist examined a subgroup of the participating young children, and the combination of answers that resulted in the highest sum of sensitivity and specificity was determined (19, $20)$. Exclusion criteria include multiple gestation (twins, triplets, etc), inability to answer questions in Chinese, and plan to move out of the area before delivery.

At $2 \mathrm{yr}$ of age, those who developed AD were gathered. We compared concentration of cord and maternal blood cotinine in AD cases and the left non-AD cases in the cohort.

\section{Questionnaire survey}

The parents were asked by home interview questionnaires at birth for birth year, parental education levels and occupation, family income, parental history of atopic diseases (such as AD, allergic rhinitis, or asthma), alcohol and drug use, diets and supplements (such as multivitamins) during pregnancy, history of smoke exposure including ETS exposure, and maternal active smoking during pregnancy. From the records of the cooperating hospitals, we gathered neonate's health data at birth, such as head circumference, birth body weight, height, weeks of gestation, and type of delivery. At $2 \mathrm{yr}$ of age, ISAAC questionnaire was performed and some postnatal exposures such as duration of breast feeding and infant formula, early consumption of egg, wheat, soy bean, or shrimp before $1 \mathrm{yr}$ of age, older siblings, furry pets or carpets at home, fungi at house walls, incensing at home, and postnatal ETS exposure were asked.

\section{Laboratory method}

The plasma samples of maternal venous blood and umbilical cord blood were stored at $-80^{\circ} \mathrm{C}$ and were sent to National Health Research Institute in Taiwan for cotinine analysis. Average $0.5 \mathrm{ml}$ of plasma was spiked with $15 \mu \mathrm{l}$ of $10 \mathrm{ng} /$ ml deuterium (D3) (ISOTEC, Miamisburg, OH, USA)-labeled cotinine (Sigma, St Louis, MO, USA) as an internal standard, $100 \mu \mathrm{l}$ of $5 \mathrm{~N}$ $\mathrm{KOH}$ (Sigma), and $3 \mathrm{ml}$ of dichloromethane (Mallinckrodt Baker, Phillipsburg, NJ, USA), followed by vigorous shaking for $1 \mathrm{~min}$ and centrifugation at $3000 \mathrm{rpm}$ for $10 \mathrm{~min}$. The clear organic phase was transferred to a tube and the aqueous phase was repeatedly extracted with dichloromethane. Then, the organic solutions were combined and dried under nitrogen. Each sample was raised in $150 \mu \mathrm{l}$ of $5 \%$ methanol (Mallinckrodt Baker) in $20 \mathrm{~mm}$ ammonium acetate (Merck, Darmstadt, Germany). Finally, $20 \mu \mathrm{l}$ was injected into high-performance liquid chromatography (PerkinElmer, Boston, MA, 
USA) coupled to a triple-quadruple tandem mass spectrometer (API 3000TM; Applied Biosystems, Foster, CA, USA) (HPLC-MS/MS) to measure the cotinine levels. The column was Purospher STAR RP-18 column (4'55 mm, $3 \mu \mathrm{m}$; MERCK, Darmstadt, Germany). The cotinine concentration was determined by comparison of the peak area to a calibration curve which was constructed by injecting nine standard cotinine concentrations ranging from 0.02 to $100 \mathrm{ng} / \mathrm{ml}$ and plotted using peak area versus the concentration of cotinine. All-tubes were deactivated. To evaluate the HPLC-MS/MS performance, a calibration curve standard was injected after every 24 samples were analyzed. The detection limit was set at $0.04 \mathrm{ng} / \mathrm{ml}$.

\section{Statistical analysis}

Cotinine concentrations were analyzed as categorical variables after division into quartiles, with the lowest quartile used as the reference category. We compared sociodemographic data of mothers and newborns in terms of cotinine levels using chi-squared tests for categorical variables and ANOVA statistics for continuous variables. The trend test was performed by chisquare tests. The relationship of cotinine levels and $\mathrm{AD}$ were estimated by means of multiple logistic regression. Analyses were performed in both univariate and multivariate models. Potential confounders from the literature reviews, including infant gender, premature birth, maternal education and occupation, alcohol consumption during pregnancy, family income, parental history of atopy, duration of breast feeding, early consumption of solid food, postnatal ETS exposure, carpets at home, and fungi on house walls were take into consideration. Only those who made $10 \%$ change in point estimate were left in the final model. All hypothesis testing was twosided at the significance level of 0.05 and performed with the SAS software version 8.2 (SAS Institute Inc., Cary, NC, USA).

\section{Results}

At $2 \mathrm{yr}$ of age, 150 mother and child pairs completed the follow-up study and specimen collection. The prevalence of most variables in those lost in the follow-up and in those completed the follow-up was not significantly different from each other. There were $38(25.3 \%)$ children developing AD. Eight (5.4\%) out of 150 mothers indicated that they smoked before this pregnancy. Two $(1.3 \%)$ out of 150 mothers smoked during pregnancy while $38(25.3 \%)$ mothers reported having ETS exposure with $0.44 \pm 1.01$ cigarettes/day. Of two mothers with active smoking during pregnancy, one $(50 \%)$ of their children developed AD. Of 38 mothers with gestational ETS exposure, eight $(21.1 \%)$ of their children had AD.

As shown in Table 1, maternal education ( $\mathrm{p}$ for trend $=0.001$ ), family income per year ( $\mathrm{p}$ for trend $=0.001$ ), maternal occupation ( $\mathrm{p}$ for trend $=0.015$ ), premature birth ( $\mathrm{p}$ for trend $=0.055)$, ETS exposure during pregnancy ( $\mathrm{p}$ for trend $=0.006$ ), and postnatal ETS exposure $(\mathrm{p}$ for trend $=0.002$ ) were found to be significantly different in the four groups on cord blood cotinine levels. Higher cotinine levels were associated with lower maternal education and family income, full-time house wives, premature birth, and ETS exposure during pregnancy and postnatal ETS exposure. The level of maternal blood cotinine was higher than that of cord blood cotinine (mean \pm s.d., $3.61 \pm 14.04 \mathrm{ng} /$ $\mathrm{ml}$ vs. $2.73 \pm 18.48 \mathrm{ng} / \mathrm{ml}, \mathrm{p}=0.642$ ). The geometric mean of maternal blood cotinine was $0.32 \mathrm{ng} / \mathrm{ml}$ while cord blood cotinine was $0.11 \mathrm{ng} / \mathrm{ml}$. The maternal and cord plasma concentrations of cotinine were highly correlated $(\mathrm{r}=0.71, \mathrm{p}<0.001)$ (Fig. 1). Table 2 revealed the association of maternal and cord blood cotinine levels with self-reported smoke exposure during pregnancy.

With respect to Table 3 , the risk of $\mathrm{AD}$ was found to increase with maternal and cord blood cotinine levels in a dose-response manner ( $\mathrm{p}$ for trend $=0.01)$. High cotinine levels $(50-75$ th percentile and $>75$ th percentile) were significantly associated with AD after adjusting for potential confounders. There was no significantly positive association between self-reported ETS exposure amount during pregnancy and $\mathrm{AD},[0$ 3 cigarettes/day vs. $3-5$ cigarettes/day; aOR 1.84 (95\% CI: $0.23-15.01)]$ and [0-3 cigarettes/day vs. $>5$ cigarettes/day; aOR 1.98 (95\% CI: $0.18-$ 19.82)] after adjusting for maternal education, maternal occupation, and postnatal ETS exposure (data not shown).

\section{Discussion}

This study is an interesting contribution to the literature on the potential association between prenatal smoke exposure and pediatric AD. To the best of our knowledge, the effect of smoke exposure during pregnancy on the development of AD in the offspring on the basis of objective biomarker has not been studied before.

Most people might be very curious about where was smoke exposure from for these 
Gestational smoke exposure and atopic dermatitis

Table 1. Characteristic of the study population according to cotinine levels

\begin{tabular}{|c|c|c|c|c|}
\hline & \multicolumn{4}{|c|}{ Cord blood cotinine levels (ng/ml) } \\
\hline & $\begin{array}{l}<0.0486 \\
(n=36)\end{array}$ & $\begin{array}{c}0.0486-0.1072 \\
(n=39)\end{array}$ & $\begin{array}{c}0.1072-0.1936 \\
(n=39)\end{array}$ & $\begin{array}{l}>0.1936 \\
(\mathrm{n}=36)\end{array}$ \\
\hline \multicolumn{5}{|l|}{ Maternal age (yr), } \\
\hline Mean \pm s.d. & $29.8 \pm 5.1$ & $30.9 \pm 5.6$ & $28.7 \pm 2.9$ & $27.6 \pm 6.0$ \\
\hline \multicolumn{5}{|l|}{ Maternal education* $(\%)$} \\
\hline High school and below & 27.8 & 38.5 & 46.2 & 86.1 \\
\hline College and above & 72.2 & 61.5 & 53.8 & 13.9 \\
\hline \multicolumn{5}{|l|}{ Maternal occupation*(\%) } \\
\hline House wives & 27.8 & 23.1 & 15.4 & 44.4 \\
\hline Non-house wives & 72.2 & 76.9 & 84.6 & 55.6 \\
\hline \multicolumn{5}{|c|}{ Maternal alcohol consumption } \\
\hline Yes $(\%)$ & 5.6 & 7.7 & 2.6 & 5.6 \\
\hline \multicolumn{5}{|c|}{ Family income per year* (NT dollars) (\%) } \\
\hline$<600,000$ & 14.3 & 15.8 & 15.4 & 50.0 \\
\hline $600,000-1,500,000$ & 65.7 & 60.5 & 59.0 & 47.2 \\
\hline$>1,500,000$ & 20.0 & 23.7 & 25.6 & 2.8 \\
\hline \multicolumn{5}{|l|}{ Infant gender $(\%)$} \\
\hline Male & 47.2 & 53.8 & 46.2 & 58.3 \\
\hline \multicolumn{5}{|l|}{ Birth weight (g) } \\
\hline Mean \pm s.d. & $3189.2 \pm 436.0$ & $3123.3 \pm 549.8$ & $3277.6 \pm 415.7$ & $3131.3 \pm 374.9$ \\
\hline \multicolumn{5}{|c|}{ Premature birth $(<37 \text { weeks })^{*}$} \\
\hline Yes $(\%)$ & 3.1 & 5.4 & 2.6 & 9.7 \\
\hline \multicolumn{5}{|l|}{ Parental history of atopy } \\
\hline Yes $(\%)$ & 22.2 & 13.2 & 28.2 & 19.4 \\
\hline \multicolumn{5}{|c|}{ Duration of breast feeding (months) } \\
\hline$<6$ & 60.0 & 69.2 & 69.2 & 74.3 \\
\hline$\geq 6$ & 40.0 & 30.8 & 30.8 & 25.7 \\
\hline \multicolumn{5}{|l|}{ Incensing at home } \\
\hline Yes $(\%)$ & 50.0 & 48.7 & 46.2 & 69.4 \\
\hline \multicolumn{5}{|c|}{ ETS exposure during pregnancy* } \\
\hline Yes $(\%)$ & 14.3 & 17.9 & 23.7 & 47.2 \\
\hline \multicolumn{5}{|l|}{ Postnatal ETS exposure* } \\
\hline Yes $(\%)$ & 25.7 & 28.2 & 23.1 & 61.8 \\
\hline \multicolumn{5}{|c|}{ Recurrent wheezing (>three episodes)* } \\
\hline Yes (\%) & 19.4 & 23.1 & 30.8 & 47.2 \\
\hline \multicolumn{5}{|l|}{ Atopic dermatitis* } \\
\hline Yes $(\%)$ & 13.9 & 20.5 & 30.8 & 36.1 \\
\hline
\end{tabular}

ETS, environmental tobacco smoke.

${ }^{*} p<0.05$.

pregnant women because most women quit smoking after they were pregnant. In our study, pregnant women had smoke exposure mainly from ETS and only trivial from active smoking. Furthermore, nicotine vapor may persist over the succeeding few days after the last cigarette was smoked in the room because of emission from contaminated room surfaces, people's clothing, or house dust (21). Surprisingly, we found ETS exposure in Taiwan was worse than that in the United States. The cotinine level in our exposed non-smokers was much higher than that reported in California (the geometric mean of maternal cotinine was $0.32 \mathrm{ng} / \mathrm{ml}$ vs. $0.08 \mathrm{ng} / \mathrm{ml}$ ) (22). Though there are regulations to restrict smoking in public and work places, ETS exposure could be obtained at home in Taiwan, where residences are often quite crowded because of limited space (23). Table 1 provides further insight into this issue. Full-time house wives had higher levels of cotinine than women with other jobs. ETS exposure was particularly serious in the room not well ventilated. These special situations in Taiwan may explain the higher cotinine levels in maternal blood in our population.

Some people might question about the objectivity of the cotinine because its measurements might be influenced by individual differences in rates or patterns of metabolism or excretion, the presence of other sources (such as nicotinecontaining food), and the sensitivity and specificity of the analytical methods. In our study, cotinine was analyzed by HPLC-MS/MS, which had excellent validity (24). In addition, recent studies indicated that the pharmacokinetics of cotinine was similar in both smokers and nonsmokers (25), suggesting that the degree of metabolism variability was likely to be similar 


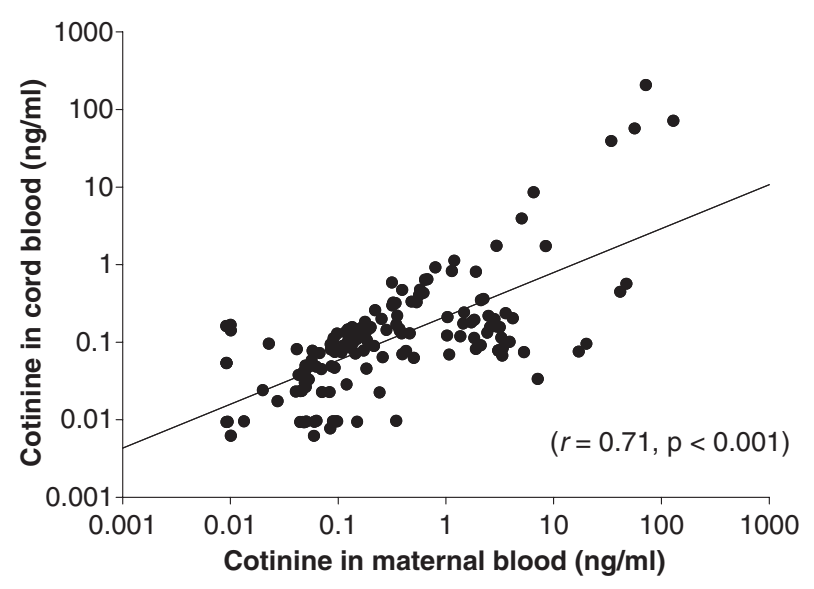

Fig. 1. The correlation of maternal and cord blood cotinine.

Table 2. The association of maternal and cord blood cotinine levels with selfreported smoke exposure during pregnancy

\begin{tabular}{lccc}
\hline & No. $(\%)$ & $\begin{array}{c}\text { Maternal blood } \\
\text { cotinine levels } \\
(\mathrm{ng} / \mathrm{ml}), \\
\text { Mean } \pm \text { s.d. }\end{array}$ & $\begin{array}{c}\text { Cord blood } \\
\text { cotinine levels } \\
(\mathrm{ng} / \mathrm{ml}), \\
\text { Mean } \pm \text { s.d. }\end{array}$ \\
\hline Total & 150 & $3.61 \pm 14.04$ & $2.73 \pm 18.48$ \\
Non-exposed non-smokers & $110(73.3)$ & $2.05 \pm 6.18$ & $0.58 \pm 3.96$ \\
Exposed non-smokers & $38(25.3)$ & $5.69 \pm 21.40$ & $5.91 \pm 30.49$ \\
Number of cigarettes/day & & & \\
$<3$ & $23(15.3)$ & $4.35 \pm 13.05$ & $0.19 \pm 0.20$ \\
$3-5$ & $8(5.3)$ & $3.91 \pm 14.73$ & $8.40 \pm 41.03$ \\
$>5$ & $7(4.7)$ & $17.58 \pm 45.15$ & $10.19 \pm 24.76$ \\
Smokers & $2(1.3)$ & $39.26 \pm 46.28$ & $106.94 \pm 139.16$ \\
\hline
\end{tabular}

for non-smokers. While nicotine is contained in some foods, such as tomatoes, potatoes, cauliflower, and black tea (26), at usual food consumption levels dietary nicotine is negligible compared with moderate ETS exposure and may not significantly confound determination of cotinine level in our study. Besides, we discovered that maternal and cord blood cotinine showed stronger association with AD than selfreported ETS exposure from our results. Based on the above reasons, we believe our exposure assessment is reliable. Data from this study provided evidence that exposure to smoking either from maternal smoking or ETS exposure during pregnancy may play a role in the development of AD. Even after adjusting for potential confounders, the risk of AD was significantly associated with cotinine levels and had doseresponse relationship. Some studies supported our observation and also demonstrated that exposure to ETS in genetically predisposed children was associated with an increased risk of sensitization to house dust mites $(11,12)$. Another study indicated that during early childhood, prenatal and postnatal ETS-exposed children had a significantly higher risk of sensitization to food allergens compared with children never exposed to ETS (27). In contrast with our study, Mills et al. found that cigarette smoking was not a risk factor for AD (14). Miyake et al. discovered that maternal smoking and household smoking in the same room as the infant were not related to the risk of AD (15). These discrepancies might be because of recall bias or different age groups. Furthermore, the possible association might have been masked by the fact that families at risk have changed their smoking habits following recent health education and prevention programs. Magnusson et al. and Linneberg et al. reported that gestational smoke exposure had mild protective effect on AD (16, 17). However, lack of the objective biomarker for smoke exposure and absence of the control options for hereditary factors in their studies may have affected the results. In addition, Noakes et al. found that there were no effects of maternal smoking on the rates of allergen sensitization and AD at 12 months of age (18). Differences of the adjusting potential confounders and the population that failed to follow up might account for the variation of the results.

If smoke exposure during pregnancy is a significant risk factor for $\mathrm{AD}$, people might be

Table 3. The association of atopic dermatitis and blood cotinine levels by univariate and multivariate logistic regression

\begin{tabular}{|c|c|c|c|c|c|}
\hline Maternal blood cotinine levels (ng/ml) & $<0.0843(n=36)$ & $0.0843-0.1920(n=39)$ & $0.1920-1.4563(n=38)$ & $>1.4563(n=36)$ & Trend test \\
\hline Crude OR $(95 \% \mathrm{Cl})$ & 1.00 & $2.76(0.78-9.76)$ & $2.86(0.81-10.13)$ & $5.09(1.48-17.53)^{*}$ & \\
\hline Adjusted OR $(95 \% \mathrm{Cl}) \dagger$ & 1.00 & $3.51(0.85-14.42)$ & $4.59(1.12-18.70)^{*}$ & $5.33(1.33-21.41)^{*}$ & $0.01^{*}$ \\
\hline Cord blood cotinine levels (ng/ml) & $<0.0486(n=36)$ & $0.0486-0.1072(n=39)$ & $0.1072-0.1936(n=39)$ & $>0.1936(n=36)$ & Trend test \\
\hline Crude OR $(95 \% \mathrm{Cl})$ & 1.00 & $1.65(0.49-5.63)$ & $2.76(0.86-8.82)$ & $3.50(1.09-11.21)^{*}$ & \\
\hline Adjusted OR $(95 \% \mathrm{Cl}) \dagger$ & 1.00 & $2.60(0.62-10.92)$ & $4.44(1.12-17.66)^{*}$ & $5.71(1.40-23.32)^{*}$ & $0.01^{*}$ \\
\hline
\end{tabular}

$\mathrm{OR}$, odds ratio; $\mathrm{Cl}$, confidence interval; ETS, environmental tobacco smoke.

${ }^{*} p<0.05$.

$\dagger$ Model adjusted for premature birth, maternal education, and postnatal ETS exposure. 
curious about how smoke enters the body of the fetus. Consistent with previous study (28), we found that there was a positive linear relationship between maternal and neonatal cotinine concentrations (Fig. 1) and the mean cotinine concentration was lower in cord blood than maternal blood in our study. This implied that cotinine, a metabolite of nicotine, used to quantify exposure to tobacco smoke, readily gained access from maternal to fetal circulation and accumulated in the fetal body. In addition, Seymour et al. reported that exposure of ovalbumin-sensitized mice to ETS by inhalation elicited a rapid and prolonged exaggerated response with respect to IgE, IgG1, eosinophils, and Th2 cytokines [particularly interleukin (IL)-4 and 1L-10] (29). Magnusson et al. discovered that cord serum $\operatorname{IgE}$ was increased in newborns if the mother smoked (13). Further evidence of altered immune responses from maternal smoking was found in a study of Australian birth cohort. It demonstrated that maternal smoking in pregnancy was associated with significantly higher neonatal Th2 (IL13 protein) responses to both house dust mite and ovalbumin (30). Sensitization to allergens along with the adjuvant effect of smoking on the Th2 response may be responsible for the prevalence of allergic symptoms seen in individuals with tobacco smoke exposure. Furthermore, tobacco smoke, like other air pollutants, may have irritant effects on skin and mucous membranes, thereby facilitating the penetration of potential allergens into the body and causing more AD symptoms and an elevated risk of sensitization (29). Then, there raised another question about which kind of ingredients in cigarettes mainly contribute to AD. The diversity of chemical ingredients found in tobacco (4000 chemicals, of which 300 were known carcinogens) was matched by the diversity of tobaccorelated health problems (31). People might suppose that nicotine was the arch criminal because it was the main constituent of tobacco. Harkavy had shown that nicotine was not the responsible antigenic component of tobacco leaf, although its role as a hapten was a possibility (32). Relevant information about the leading element in cigarettes to AD, however, was not available. Further studies are warranted.

The strengths of our study included the population-based prospective cohort design and objective biomarkers of smoking exposure. Cotinine was a valid quantitative predictor of the level of smoke exposure for population studies. However, because the smoke exposure was intermittent and cotinine was a biomarker of recent exposure with a half-life of approximately
$20 \mathrm{~h} \mathrm{(6),} \mathrm{one} \mathrm{of} \mathrm{our} \mathrm{limitations} \mathrm{was} \mathrm{that} \mathrm{the}$ cotinine measurement from cord blood was a single opportunistic measurement at delivery. In our study, cord blood cotinine level revealed a good correlation to self-reported smoke exposure amount (Table 3) and smoking habits investigated in our questionnaire were relatively stable over time. According to previous study, cotinine levels remained fairly constant and at near steady-state values throughout the day (6). Furthermore, if measurement error did occur, it tended to be toward the null and the effect of exposure was likely to be underestimated. Another limitation was that there is probably a considerable overlap between pregnancy and postnatal exposure and therefore also an association between cotinine levels and postnatal exposure, which makes it very difficult to disentangle the relevance of the different time windows. As the correlation and the collinearity of pregnancy and postnatal ETS exposure was low $(\mathrm{r}=0.3, \mathrm{p}=0.06)$ in our study, we put postnatal ETS exposure into the model as a potential confounder. Levels of cord cotinine concentration were still positively related to development of AD even after adjusting for postnatal ETS exposure. The other limitation was that a potential selection bias could have occurred if non-participants were different on sociodemographic or smoking characteristics from participants. However, the prevalence of most variables in those lost in the follow-up and in those completed the follow-up was not significantly different from each other. If a selection bias would occur, it might be trivial.

In conclusion, we observe that ETS exposure in Taiwan is more serious than expected. Our study supports the hypothesis that ETS exposure and maternal smoking during pregnancy might be a risk factor for pediatric AD. Avoidance of prenatal smoke exposure should be paid more attention.

\section{Acknowledgments}

This study was supported in part by grants from the Bureau of Health Promotion, Department of Health (BHP-PHRC92-4 and DOH93-HP-1702), and the National Science Council (NSC95-2314-B-002-269 and NSC96-2314-B-192001) of Taiwan.

\section{References}

1. Martinez FD, Cline M, Burrows B. Increased incidence of asthma in children of smoking mothers. Pediatrics 1992: 89: 21-6.

2. Evans D, Levison MJ, Feldman CH, et al. The impact of passive smoking on emergency room visits of urban children with asthma. Am Rev Respir Dis 1987: 135: 567-72. 
3. Perez-Stable EJ, Benowitz NL, Marin G. Is serum cotinine a better measure of cigarette smoking than selfreport? Prev Med 1995: 24: 171-9.

4. DeLorenze GN, Kharrazi M, Kaufman FL, EskenAZI B, BERnERT JT. Exposure to environmental tobacco smoke in pregnant women: the association between selfreport and serum cotinine. Environ Res 2002: 90: 21-32.

5. BenOwITZ NL. Biomarkers of environmental tobacco smoke exposure. Environ Health Perspect 1999: 107: 349-55.

6. Benowitz NL, Jacob P III. Metabolism of nicotine to cotinine studied by a dual stable isotope method. Clin Pharmacol Ther 1994: 56: 483-93.

7. Nafstad P, Kongerud J, Botten G, et al. Fetal exposure to tobacco smoke products: a comparison between self-reported maternal smoking and concentrations of cotinine and thiocyanate in cord serum. Acta Obstet Gynecol Scand 1996: 75: 902-7.

8. Pichini S, Basagana XB, Pacifici R, et al. Cord serum cotinine as a biomarker of fetal exposure to cigarette smoke at the end of pregnancy. Environ Health Perspect 2000: 108: 1079-83.

9. Wen CP, Levy DT, Cheng TY, Hsu CC, Tsai SP. Smoking behavior in Taiwan. Tob Control 2001: 14: i51-5.

10. Kharrazi M, Delorenze GN, Kaufman FL, et al. Environmental tobacco smoke and pregnancy outcome. Epidemiology 2004: 15: 660-70.

11. Krämer U, Lemmen CH, Behrend H, et al. The effect of environmental tobacco smoke on eczema and allergic sensitization in children. Br J Dermatol 2004: 150: 111-8.

12. Schafer T, Dirschedl P, Kulz B, Ring J, Uberla K. Maternal smoking during pregnancy and lactation increases the risk for atopic eczema in the offspring. J Am Acad Dermatol 1997: 36: 550-6.

13. Magnusson CG. Maternal smoking influences cord serum $\operatorname{IgE}$ and $\mathrm{IgD}$ levels and increases the risk for subsequent infant allergy. J Allergy Clin Immunol 1986: 78: 898-904.

14. Mills CM, Srivastava ED, Harvey IM, et al. Cigarette smoking is not a risk factor in atopic dermatitis. Int J Dermatol 1994: 33: 33-4.

15. Miyake Y, Ohya Y, Tanaka K, et al. Home environment and suspected atopic eczema in Japanese infants: The Osaka Maternal and Child Health Study. Pediatr Allergy Immunol 2007: 18: 425-32.

16. Magnusson Ll, Olesen AB, Wennborg H, Olsen J. Wheezing, asthma, hayfever, and atopic eczema in childhood following exposure to tobacco smoke in fetal life. Clin Exp Allergy 2005: 35: 1550-6.

17. Linneberg A, Simonsen JB, Petersen J, Stensballe LG, BENN CS. Differential effects of risk factors on infant wheeze and atopic dermatitis emphasize a different etiology. J Allergy Clin Immunol 2006: 117: 184-9.

18. Noakes P, Taylor A, Hale J, et al. The effects of maternal smoking on early mucosal immunity and sensitization at 12 months of age. Pediatr Allergy Immunol 2007: 18: 118-27.
19. Chan HH, Pei A, Van Krevel C, Wong GWK, Lai CKW. Validation of the Chinese translated version of ISAAC core questions for atopic eczema. Clin Exp Allergy 2001: 31: 903.

20. Benn CS, Benfeldt E, Andersen PK, Olesen AB, Melbye M, Bjorksten B. Atopic dermatitis in young children: diagnostic criteria for use in epidemiological studies based on telephone interviews. Acta Derm Venereol 2003: 83: 347-50.

21. Nelson PR, DeBethizy JD, Davis RA, et al. Where there's smoke? Biases in the use of nicotine and cotinine as environmental tobacco smoke biomarkers. Proceedings of the 1991 Environmental Protection Agency/Air and Waste Management Association International Symposium: Measurement of Toxic and Related Air Pollutants. Pittsburgh, PA: Air and Waste Management Association, 1991: 449-54.

22. Pirkle Jl, Flegal KM, Bernert JT, Brody DJ, Etzel RA, Maurer KR. Exposure of the US population to environmental tobacco smoke: the Third National Health and Nutrition Examination Survey, 1988 to 1991. JAMA 1996: 275: 1233-40.

23. Government Information Office, Taiwan. http:// www.gio.gov.tw/info/taiwan-story/society/down/3-4.htm.

24. JАCOB P III, Yu L, Wilson M, Benowitz NL. Selected ion monitoring method for determination of nicotine, cotinine, and deuteriumlabeled analogs: absence of an isotope effect in the clearance of (S)-nicotine- $3^{\prime}-3^{\prime}-\mathrm{d} 2$ in humans. Biol Mass Spectrom 1991: 20: 247-52.

25. Benowitz NL, JACOB P III. Nicotine and cotinine elimination pharmacokinetics in smokers and nonsmokers. Clin Pharmacol Ther 1993: 53: 316-23.

26. Davis RA, Stiles MF, DeBethizy JD, Reynolds JH. Dietary nicotine: a source of urinary cotinine. Food Chem Toxicol 1991: 29: 821-7.

27. KUlig M, LUCK W, WAHN U. The association between pre- and postnatal tobacco smoke exposure and allergic sensitization during early childhood. Hum Exp Toxicol 1999: 18: 241-4.

28. Donnenfeld AE, Pulkkinen A, Palomaki GE, Knight GJ, Haddow JE. Simultaneous fetal and maternal cotinine levels in pregnant women smokers. Am J Obstet Gynecol 1993: 168 (3 Pt 1): 781-2.

29. Seymour BW, Pinkerton KE, Friebertshauser KE, Coffman RL, Gershwin LJ. Second hand smoke is an adjuvant for $\mathrm{T}$ helper-2 responses in a murine model of allergy. J Immunol 1997: 159: 6169-75.

30. Noakes PS, Holt PG, Prescott SL. Maternal smoking in pregnancy alters neonatal cytokine responses. Allergy 2003: 58: 1053-8.

31. Roemer E, Stabbert R, Rustemeier K, et al. Chemical composition, cytotoxicity and mutagenicity of smoke from US commercial and reference cigarettes smoked under two sets of machine smoking conditions. Toxicology 2004: 951: 31-52.

32. Harkavy J. Tobacco allergy in cardiovascular disease: a review. Ann Allergy 1968: 26: 447-59. 